\title{
Follow-up Study Comparing Open Hysterectomy of Expert Surgeon and Laparoscopic Approach (Learning Curve) of the Same Surgeon
}

\author{
Maliheh Arab ${ }^{1}$, Rajneesh K Mishra ${ }^{2}$, Jatinder Sigh Chowhan ${ }^{3}$, Shahla N Ardebili ${ }^{4}$, Behnaz Ghavami ${ }^{5}$, Nasrin Yousefi ${ }^{6}$
}

\begin{abstract}
Introduction: The goal of minimal access surgery is to minimize damage to the patient without impairment of immunity and the effect of treatment compared to traditional open surgical techniques. Laparoscopic hysterectomy requires more surgical skills and the learning curve is steep. The goal of this study is to compare hysterectomy in learning curve (including about 50 first surgeries) with open hysterectomy of the same surgeon, expert in open surgery, for complications, hospital stay duration, transfusion, operative time, and readmission.

Materials and methods: In a prospective cohort study, patients undergoing hysterectomy at an academic medical center located in Tehran were randomly assigned into laparoscopic (in learning curve) and laparotomy groups from 2016 to 2018. Study cases data were recorded regarding complications, hospital stay, operative time, and blood transfusion.

Results: There was no significant difference regarding intra- and postoperative transfusion, hospital stay duration, postoperative complications, and readmission in laparoscopy and laparotomy groups of hysterectomy. However, operative time was significantly different in laparoscopy and laparotomy subgroups of hysterectomy and longer in the laparoscopic group ( 277 minutes in laparoscopy vs 196 minutes in laparotomy). Conclusion: This study encourages starting laparoscopy method instead of open surgery, even in the setting of expert open surgeons, and even in the advanced (level 4) surgery such as hysterectomy.

Keywords: Complications, Hysterectomy, Laparoscopy, Laparotomy, Learning curve.

World Journal of Laparoscopic Surgery (2019): 10.5005/jp-journals-10033-1359
\end{abstract}

\section{INTRODUCTION}

The goal of minimal access surgery is to minimize damage to the patient without impairment of immunity and the effect of treatment compared to traditional open surgical techniques. If this goal is achieved, patients will recover faster, and hospitalization will be reduced, and their return to full activity and work will be returned in a short time. ${ }^{1-3}$ The history of laparoscopy is still short and still no long-term results in comparison to open surgery are in our hands. ${ }^{4}$ Today there is a lot of evidence of laparoscopic preference, and they all accept it. ${ }^{5}$ In general, laparoscopic complications are less than open surgery. ${ }^{6}$

In 1999, laparoscopic hysterectomy was considered an alternative for open surgery. The first laparoscopy was reported in 1989, and then, this method continued. In the case of laparoscopic hysterectomy, compared with open surgery, the surgical time is significantly longer. ${ }^{7,8}$ In a study, the time of postoperative recovery and the pain score in 37 patients with primary pelvic pain with diagnosis of fibroma, adenomyosis, and severe endometriosis who underwent laparoscopic assisted vaginal hysterectomy (LAVH) were recorded. The length of hospitalization was 4.5 and 2.5 days after open hysterectomy and LAVH, respectively. LAVH is more expensive than total abdominal hysterectomy (TAH). The issue is whether the benefits of shorter recovery and faster return to work, shorter hospitalization, and less need for pain relief cover the extra cost of laparoscopy. If the total healthcare costs are evaluated, the short-term recovery of laparoscopy, 2 weeks, compared to the recovery of 6-8 weeks after open surgery, makes it costly. LAVH can replace most abdominal hysterectomies due to benign disease. Laparoscopic hysterectomy requires more surgical skills, and the learning curve is steep. Studies have shown that laparoscopic advantages comparing to laparotomy
'Department of Minimal Access Surgery, Cancer Research Center, Shahid Beheshti University of Medical Sciences, Tehran, Iran

${ }^{2}$ Department of Minimal Access Surgery, The Global Open University, Dimapur, Nagaland, India

${ }^{3}$ Department of Minimal Access Surgery, World Laparoscopy Hospital, Gurugram, Haryana, India

${ }^{4,6}$ Department of Obstetrics and Gynaecology, Shahid Beheshti University of Medical Sciences, Tehran, Iran

${ }^{5}$ Department of Minimal Access Surgery, Tehran University of Medical Sciences, Tehran, Iran

Corresponding Author: Maliheh Arab, Department of Minimal Access Surgery, Cancer Research Center, Shahid Beheshti University of Medical Sciences, Tehran, Iran, Phone: +91 9811416838, e-mail: drmarab@yahoo.com

How to cite this article: Arab M, Mishra RK, Chowhan JS, et al. Followup Study Comparing Open Hysterectomy of Expert Surgeon and Laparoscopic Approach (Learning Curve) of the Same Surgeon. World J Lap Surg 2019;12(1):5-8.

Source of support: Nil

Conflict of interest: None

include reduced postoperative pain, shorter hospitalization, faster recovery, and faster social recovery. Laparoscopic hysterectomy is longer in all studies. ${ }^{1,9,10}$

\section{Training in Laparoscopy}

Besides the great interest in laparoscopy, the cost of training and instruments increase the total cost. On the other hand, less

() The Author(s). 2019 Open Access This article is distributed under the terms of the Creative Commons Attribution 4.0 International License (https://creativecommons. org/licenses/by-nc/4.0/), which permits unrestricted use, distribution, and non-commercial reproduction in any medium, provided you give appropriate credit to the original author(s) and the source, provide a link to the Creative Commons license, and indicate if changes were made. The Creative Commons Public Domain Dedication waiver (http://creativecommons.org/publicdomain/zero/1.0/) applies to the data made available in this article, unless otherwise stated. 
complications and rapid recovery of laparoscopy cover these shortages. ${ }^{4}$ Three characters of laparoscopy are as follows: instruments, trained personnel, and learning curve. ${ }^{11}$ Nowadays, surgeons work on basic skills before real surgery. ${ }^{12}$

Learning curve includes 3 phases, starting, learning rate, and stabilized performance. The speed of laparoscopic learning curve at first phase is not dependent on age, number of surgeries, or hospital setting. The first phase is rapid. The main factor effective on learning curve is the supporting surgical team. Another factor is the equipment problem which is reported to occur in $87 \%$ of procedures. ${ }^{5,13-15}$

Learning curve is defined by the number of patients which reduce complications and time of surgery toward the same procedure in the open method. During the learning curve, complications are higher and the operative time is longer. Learning curve is defined in difficult procedures, for instance, in appendectomy, learning curve is about 30 patients. ${ }^{5,6,16-18}$

\section{Levels of Gynecological Laparoscopic Surgery (HKCOG)}

Level 1: Basic procedures such as diagnostic and tubal occlusion Level 2: Minor procedures such as salpingectomy for tubal pregnancy or hydrosalpinx

Level 3: Intermediate procedures such as oophorectomy or cystectomy for ovarian cysts

Level 4: Major procedures such as hysterectomy and myomectomy Level 5: Advanced procedures such as lymphadenectomy and radical hysterectomy

In this study, we compare hysterectomy in learning curve (including about 50 first surgeries) with open hysterectomy of the same surgeon, expert in open surgery, for complications, hospital stay duration, transfusion, operative time, and readmission.

\section{Materials and Methods}

\section{Study Area and Study Population}

In a prospective cohort study, patients undergoing hysterectomy at the Imam Hossein Medical Center located in Tehran were randomly assigned into laparoscopic and laparotomy groups from 2016 to 2018.

In this study, surgeon was the same in all operations. It should be noted that the surgeon's work experience in open surgery was about 20 years, and she was an expert, a radical gynecooncologist, and a referral of difficult surgical procedures. The above-mentioned surgeon began to perform laparoscopy in hysterectomy by participating in 3 laparoscopic workshops and using a trainer for a period of 6 months and clinical practice with an expert laparoscopist for 6 months, mostly in level 3 operations; finally participated in the one-month compact laparoscopy course again and started laparoscopic hysterectomy operations (level 4), independently.

From the beginning, under study information, cases were recorded regarding complications, hospital stay, operative time, and blood transfusion.

\section{Surgical Techniques}

The patient was placed in the lithotomy position with her legs open at $60^{\circ}$, under general anesthesia with endotracheal intubation; a Foley urinary catheter ensured the bladder was emptied during the operation.

After a $\mathrm{CO}_{2}$ pneumoperitoneum was created, a $10-\mathrm{mm}$ trocar was placed in the umbilical site by the modified Hasson technique to introduce the laparoscope and the camera. Three ancillary 5 - $\mathrm{mm}$ trocars were also placed, two in the left side $(7 \mathrm{~cm}$ apart to each other) and one in the right side of the patient. The surgeon operated ipsilaterally and her assistant worked in contralateral side and handled the camera at the same time.

The round ligament was sectioned at $\sim 3 \mathrm{~cm}$ from the uterus, by Harmonic Ace in order to prevent bleeding from the superior uterine vessels. The areolar tissue of the broad ligament was then dissected and its posterior fold fenestrated at an avascular area above the uterine vessels. The uterine artery and the utero-ovarian ligament vs infundibulo-pelvic ligament in both sides were tied by suture and cut by Harmonic Ace.

After complete dissection of the bladder, circular monopolar colpotomy was then performed, and the uterus was removed through the vagina and sent for histological examination.

At this stage, the uterine manipulator was extremely effective in completely exposing the fornices and at the same time in avoiding $\mathrm{CO}_{2}$ leakage from the pneumoperitoneum, thus making colpotomy easier. Finally, the vaginal vault was sutured continuously laparoscopically, and the pelvis was then checked in order to ensure hemostasis and to perform pelvic irrigation, thus removing blood clots. At the end of the surgery, only fascia site of $10 \mathrm{~mm}$ trochars was repaired. In the open surgery, hemostasis was performed by electrocautery and suturing, and in the case of hysterectomy, the vaginal cuff was closed.

The beginning of the operation was calculated as the moment of the umbilical incision and for laparoscopic hysterectomy and as the moment of cutaneous incision for the abdominal technique. Cutaneous suture was considered the end of the operation in both cases.

\section{Sample Size}

Cases of hysterectomy were divided into 54 laparoscopy and 57 laparotomy method. Laparoscopy cases were considered in the learning curve group. So, there were two groups of hysterectomy, including laparoscopy (learning) and laparotomy.

\section{Data Collection}

Complications during hospital stay and after discharge, blood transfusion, duration of hospitalization, readmission, and the surgical time of patients were compared between two groups.

\section{Statistical Method}

The normal distribution of quantitative data was performed using Shapiro-Wilk test. Quantitative data were displayed using mean, standard deviations, mid-range, and interquartile domains. The qualitative data were displayed using frequency and percent. Data were analyzed by ANOVA, Kruskal-Wallis, T-independent, Mann-Whitney, and Kendall-Tau coefficients for comparing quantitative responses between groups. Guerrilla post hoc test was used whenever necessary. Chi-square test was used to compare the qualitative responses between the studied groups, and if necessary, the exact $p$ value was calculated. Covariance analysis was used to compare postoperative hemoglobin between the studied groups. The significance level for statistical tests was considered 0.05. SPSS software version 25 was used for data analysis.

\section{Results}

A total of 111 patients underwent hysterectomy. In the hysterectomy group, 111 patients, including laparoscopy in learning curve group (54) and laparotomy (57), were studied. 
Medical disease, mean age, and preoperative hemoglobin level were not significantly different in patients under 2 groups of laparotomy and laparoscopy (Table 1).

There was no significant difference regarding intra- and postoperative transfusion, hospital stay duration, postoperative complications, and readmission in laparoscopy and laparotomy groups of hysterectomy. However, the operative time was significantly different in laparoscopy and laparotomy subgroups of hysterectomy, longer in the laparoscopic group ( 277 minutes in laparoscopy vs 196 minutes in laparotomy) (Table 2).

The type of complications during hospital stay and longterm and total complications were not significantly different in laparoscopy and laparotomy groups of hysterectomy $(p=0.5)$. No major complications happened in each of two groups.

No case of conversion to laparotomy existed in the studied laparoscopy cases.

\section{Discussion}

\section{Transfusion and Blood Loss}

In the present study, transfusion during and after surgery did not differ significantly between the laparoscopy and laparotomy groups. ${ }^{19}$ In the other hand, in the present study, just the outcome of blood transfusion was compared in 2 groups and the volume of blood loss was not measured. Probably, if it was done, the difference of blood loss volume might be different in 2 methods. In addition to the experience of the surgeon, the staffing issues and the surgeon's assistant also play a role in the outcome of laparoscopy including blood loss.

Table 1: Comparison of demographic data, underlying medical disease, and preoperative hemoglobin level in laparoscopic and laparotomy groups of hysterectomy surgery

\begin{tabular}{llll}
\hline \multirow{2}{*}{ Variables } & \multicolumn{3}{l}{ Group } \\
\cline { 2 - 4 } & Laparoscopy & Laparotomy & $p$ \\
\hline Mean age (SD) & $46.37(6.8)$ & $47.7(7)$ & 0.318 \\
Medical disease, $n$ (\%) & $35 / 54(64.8)$ & $34 / 56(60.7)$ & 0.657 \\
Mean BMI (SD) & $28.18(4.7)$ & $28.59(5.7)$ & 0.712 \\
Mean preoperative Hb (SD) & $11.57(1.76)$ & $11.34(1.94)$ & 0.516 \\
\hline
\end{tabular}

Table 2: Comparison between laparoscopy and laparotomy groups of hysterectomy surgery regarding different variables

\begin{tabular}{llll}
\hline & \multicolumn{3}{l}{ Group } \\
\cline { 2 - 4 } Variables & Laparoscopy & Laparotomy & $p$ \\
\hline $\begin{array}{l}\text { Intraoperative transfusion, } \\
n(\%)\end{array}$ & $3 / 54(5.6)$ & $5 / 57(8.9)$ & 0.999 \\
$\begin{array}{l}\text { Postoperative transfusion, } \\
n(\%)\end{array}$ & $8 / 54(14.9)$ & $5 / 57(8.8)$ & 0.225 \\
Mean operative time (SD) & $277.44(84.48)$ & $196.75(62.13)$ & 0.005 \\
Mean hospital stay (SD) & $2.59(1.22)$ & $2.7(1.08)$ & 0.211 \\
Hospital stay complications & $10 / 54(18.5)$ & $4 / 57(7)$ & 0.68 \\
$\begin{array}{l}\text { Long-term complications } \\
\text { Total postoperative }\end{array}$ & $12 / 54(22.2)$ & $7 / 57(12.3)$ & 0.51 \\
complications & $17 / 54(31.5)$ & $9 / 57(15.8)$ & 0.51 \\
Rehospitalization, $n(\%)$ & $1 / 54(1.9)$ & $1 / 57(1.8)$ & 0.999 \\
\hline
\end{tabular}

\section{Operation Time}

In the present study, the surgical time of the two groups had significant difference (277 minutes in laparoscopy vs 196 minutes in laparotomy). In some studies, laparoscopic and open hysterectomy were compared, and the learning curve was investigated in a prospective study and there was no difference in complications. ${ }^{7,19,20}$ In a study, the operating time of laparoscopic history was $104 \pm 26$ minutes, and after passing the learning curve, it was 72-163 minutes with no significant difference with open surgery. ${ }^{7}$ An important point is different reports of early years of laparoscopy with longer procedures in comparison to the open method. ${ }^{20}$

Three characters are regarded for learning curve assessment including the duration of surgery, rate of complications, and the number of conversions to open surgery. In a study, in the learning curve of laparoscopic hysterectomy, the first 10 procedures were done in a mean time of 180 minutes and decreased to 75 minutes in the 90-100th patients. ${ }^{21}$

In the medical center of the present study, the nursing staff, equipment, and engineering were also in training period (learning curve), and the effect of these factors was also evident in the operative time. For instance, unchecked instruments, camera, and monitoring system exhibited problems during operation which took time to solve each of them. Of course, whenever the working system develops, less problems occur during operation, and if happens, solution is rapidly done.

\section{Complications}

In the present study, complications during hospitalization and longterm (after discharge) and total complications of surgery were not significantly different between the two groups of laparoscopy and laparotomy. No serious complications occurred in two groups, and the readmission of the two groups did not differ.

Considering that the surgeon was expert in the open surgery and radical operations, the complications of her open surgery were less. The point that complications of the open surgery group with a 20-year experience of surgeon and laparoscopic surgery in her learning curve did not have a significant difference is in favor of confirming less complications of laparoscopic surgery.

\section{Hospital Stay}

In a study of laparoscopic and open hysterectomy, the mean length of the hospital stay was $2.38 \pm 0.30$ days in the laparoscopic hysterectomy group vs $6.23 \pm 1.85$ days in the abdominal hysterectomy group $(p \leq 0.001){ }^{7}$

In the present study, the hospital stay was not different in two groups of laparoscopy and laparotomy. However, patients were not discharged, even if they wanted and were ready to leave hospital, given that the surgeon noted that she was in learning curve and was willing to closely observe postoperative period of laparoscopy patients. In this study, the need for patient pain relief, comfort, satisfaction, and quicker return to work were not considered, which might be better in the laparoscopic group.

\section{Readmission}

In the present study, readmission was not different in two groups.

\section{Conversion Rate}

In a study, readmission rate and complication rate of laparoscopic colorectal surgery were not different in comparison to expert surgeons, although decrease in operative time and conversion rate 
was demonstrated. These finding might be due to more complex and high risk patients accepted by expert surgeons. Another study confirmed that the effect of change in the character of patients, tendency of complicated cases accepted by expert surgeons. ${ }^{17}$

The main reason for the conversion rate is usually a complication. So, conversion and complication rate are more in learning curve in the present study. There was no case of conversion to open surgery in laparoscopy patients.

\section{Learning Curve}

Transfusion

In the present study, transfusion during and after surgery did not differ significantly between the laparoscopy and laparotomy groups. In the laparoscopic surgery, blood loss is expected to be less than open surgery. A study in the laparoscopic and laparotomy hysterectomy showed that bleeding during laparoscopic surgery was less than open surgery $(p<0.001)$. The average intraoperative blood loss was lower in laparoscopic hysterectomy than in abdominal hysterectomy $(p \leq 0.001) .^{17}$

\section{Conclusion}

In the present study, hysterectomy patients were operated on in two groups of laparoscopy (learning curve) and open surgery of expert and radical surgeon, which did not differ in terms of complications, transfusion, duration of hospitalization, and readmission. However, the surgical time was significantly longer in the laparoscopy group. This study encourages starting laparoscopy method instead of open surgery, even in setting of expert open surgeons, and even in advanced (level 4) surgery such as hysterectomy.

In the present study, the surgeon was a gyneco-oncologist and was very familiar to pelvic anatomy and an expert in open surgery. Probably, equal complication, transfusion, hospital stay, and readmission of laparoscopic hysterectomy in her learning curve in comparison to her open surgery were due to prolonged experience in radical surgeries and might not be the case of every open surgeon.

Another point is no attention and data collection regarding patient satisfaction with her operation and work return delay after each method of surgery, laparoscopy and open, which are the main advantages of laparoscopic surgery.

\section{References}

1. Mishra RK. Text book of Practical Laparoscopic Surgery. In: Mishra RK. ed. Chronological Advances in Minimal Access Surgery, 3rd ed., New Delhi: Jaypee Brothers Medical Publisher (P) Ltd; 2013. pp. 3-7.

2. Hurd W, Rivlin ME. Gynecologic Laparoscopy. emedicine.medscape. com. Sep 12, 2018.

3. Gunning JE. The history of laparoscopy. J Reprod Med 1974;12: 222-225.
4. Mán E, Németh T, et al. Learning curve after rapid introduction of laparoscopic appendectomy: are there any risks in surgical resident participation? World J Emerg Surg 2016. DOI: 10.1186/s13017-0160074-5.

5. Raja RJ. The Impact of the Learning Curve in Laparoscopic Surgery. World J Laparoscopic Surg 2008;1(1):56-59. DOI: 10.5005/ jp-journals-10007-1048.

6. Terzi H, Biler A, et al. Total laparoscopic hysterectomy: Analysis of the surgical learning curve in benign conditions. Int J Surg November 2016;35:51-57. DOI: 10.1016/j.jijsu.2016.09.010.

7. Perino A, Cucinella G, et al. Total laparoscopic hysterectomy versus total abdominal hysterectomy: an assessment of the learning curve in a prospective randomized study. Human Reprod 1999;14:2996-2999. DOI: 10.1093/humrep/14.12.2996.

8. LirkP, Thiry J, et al. Pain management after laparoscopic hysterectomy: systematic review of literature and PROSPECT recommendations. BMJ Feb 2019. DOI: 10.1136/rapm-2018-100024.

9. Chapron C, Dubuisson JB, et al. Total laparoscopic hysterectomy: preliminary results. Hum Reprod 1994;9:2084-2089. DOI: 10.1093/ oxfordjournals.humrep.a138398.

10. Chapron C, Fauconnier A, et al. Laparoscopic surgery is not inherently dangerous for patients presenting with benign gynaecologic pathology. Results of a meta-analysis. Hum Reprod 2002;17: 1334-1342. DOI: 10.1093/humrep/17.5.1334.

11. www.hkcog.org.hk/hkcog/Download/endo_levels_July2006.pdf.

12. Mishra RK. Text book of Practical Laparoscopic Surgery. In: Mishra RK. ed. Role of Training in Minimal Access Surgery, 3rd ed., New Delhi: Jaypee Brothers Medical Publisher (P) Ltd; 2013. pp. 532-539.

13. Papachristofi $O$, Jenkins $D$, et al. Assessment of learning curves in complex surgical interventions: a consecutive case-series study. BMS Series June 2016. DOI: 10.1186/s13063-016-1383-4.

14. Michel LA. Epistelogy of evidence-based medicine. Surg Endosc 2007;21(2):146. DOI: 10.1007/s00464-006-0905-7.

15. Verdaasdonk EGG, Stassen LPS, et al. Problems with technical equipment during laparoscopic surgery. Surg Endosc 2007;21: 275-279. DOI: 10.1007/s00464-006-0019-2.

16. Mavrona R, Radosa JC, et al. Learning curves for laparoscopic hysterectomy after implementation of minimally invasive surgery. Researchgate May 2016.

17. Bozkurt N, Yildiz S, et al. Total Laparoscopic Hysterectomy: Learning Curve of a Single Surgeon with 40 Cases. The journal of minimally invasive Gynecology. November-December, 2013;20(6):S150.

18. Graves N, Janda M, et al. The cost-effectiveness of total laparoscopic hysterectomy compared to total abdominal hysterectomy for the treatment of early stage endometrial cancer. J Health Econ 2012.

19. Marana R, Busacca M, et al. Laparoscopically assisted vaginal hysterectomy versus total abdominal hysterectomy: a prospective, randomized, multicenter study. Am J Obstet Gynecol 1999;180: 270-275. DOI: 10.1016/S0002-9378(99)70199-7.

20. MacKoul P, Danilyants N, et al. Laparoscopic Hysterectomy Outcomes: Hospital vs Ambulatory Surgery Center. JSLS 2019 JanMar;23(1):e2018.00076. DOI: 10.4293/JSLS.2018.00076.

21. Torng PL, Pan SP, et al. Learning curve in concurrent application of laparoscopic and robotic-assisted hysterectomy with lymphadenectomy in endometrial cancer. Taiwan J Obstet Gynecol December 2017;56(6):781-787. DOI: 10.1016/j.tjog.2017.10.014. 